\title{
Efecto del calibre de la aguja sobre la eficacia de la hemodiálisis
}

\author{
Amalia J arriz Mondueri \\ Elsa Bardales Calero \\ Almudena Pulido Arce \\ Marta Albalate Ramón
}

Fundación Renal Iñigo Álvarez de Toledo

Centro de Diálisis "Santa Engracia"

\section{RESUMEN}

Uno de los factores íntimamente relacionados con el aclaramiento de solutos durante la HD es el flujo de sangre; para que éste sea el adecuado es importante considerar el calibre de la aguja.

Estudiamos el efecto de los cambios del calibre de la aguja sobre la eficacia de la DH considerando el tipo de acceso vascular. Se diseñó un estudio prospectivo en 66 pacientes en HD (48 con fístula nativa y 18 con protésica), en los que se modificó el calibre de la aguja. Se registró dolor, sangrado, tiempo de coagulación, presión venosa y KT/v.

El uso de agujas $15 \mathrm{G}$ en la punción arterial mejora el aclaramiento de urea en las fístulas nativas pero no lo hace en las protésicas. El uso de agujas $14 \mathrm{G}$ provoca más episodios de dolor, sangrado periaguja y prolongación del tiempo de coagulación, por lo que recomendamos el uso de agujas $15 \mathrm{G}$ para conseguir una mayor eficacia en la HD a flujos de $350 \mathrm{ml} / \mathrm{min}$.

PALABRAS CLAVE: CALIBRE AGUJA

FLUJ O SANGUÍNEO

HEMODIÁLISIS

ACCESO VASCULAR

\section{EFFECT OF THE NEEDLE CALIBRE ON THE EFFICIENCY OF HAEMODIALYSIS}

\section{ABSTRACT}

O ne of the factors closely related to the clearance of solutes during HD is the blood flow; in order for the blood flow to be adequate, it is important to consider the needle calibre.

We studied the effect of changes in needle calibre on the efficiency of HD, considering the type of vascular access. A prospective study in 66 HD patients was designed (48 with native fistula and 18 with con prosthetic), in which the needle calibre was modified. Pain, bleeding, coagulation times, vein pressure and $\mathrm{Kt} / \mathrm{V}$ were recorded.

The use of $15 \mathrm{G}$ needs in the arterial puncture improves the clearance of urea in native fistulas but not in the prosthetic fistulas. The use of $14 \mathrm{G}$ needles causes more episodes of pain, bleeding around the puncture site and prolongation of the coagulation time, and therefore we recommend the use of $15 \mathrm{G}$ needles to achieve greater efficiency in HD at flows of $350 \mathrm{ml} / \mathrm{min}$.

KEYWORDS:

$$
\begin{aligned}
& \text { NEEDLE CALIBRE } \\
& \text { BLOOD FLOW } \\
& \text { HAEMODIALYSIS } \\
& \text { VASCULAR ACCESS }
\end{aligned}
$$

\section{INTRODUCCIÓN}

Uno de los factores íntimamente relacionado con el aclaramiento de solutos en hemodiálisis (HD) es el flujo de sangre ${ }^{1,2}$. Aunque el aumento del aclaramiento sanguíneo no es directamente proporcional al mayor flujo de sangre por la interferencia de otros factores, la mayoría de las unidades de diálisis 
prescriben flujos de sangre pautados mayores a $300 \mathrm{ml} / \mathrm{min}$, que todos sabemos son superiores al flujo real. Para que éste sea el que nos indica la bomba es importante utilizar un calibre de aguja apropiado que permita alcanzar dichos flujos y disminuir la recirculación, logrando así la máxima eficacia depuradora de toxinas. No está suficientemente aclarado en la bibliografía si dicho beneficio teórico es igual en accesos vasculares autólogos o protésicos, dado que distintos estudios realizados sólo se incluyen fístulas nativas o bien no se indica el tipo de acceso vascular del paciente ${ }^{3-6}$.

\section{OBJETIVOS}

1) Estudiar el efecto de los cambios de calibre de la aguja arterial y venosa sobre la eficacia de la HD.

2) Observar la posible influencia del tipo de acceso vascular en el resultado.

3) Recoger otros efectos del cambio del calibre de la aguja sobre el dolor de la punción, el sangrado periaguja y el tiempo de coagulación.

\section{MATERIAL Y MÉTODOS}

Se evaluaron 82 pacientes en tratamiento con HD en nuestro centro, de los que 16 fueron excluidos del estudio por diversos motivos (ingresos hospitalarios, HD por catéteres centrales y trasplante renal). Así, se incluyeron 66 pacientes (39 hombres y 27 mujeres) con una edad media de 65 años (24-87 años).

Todos recibían tratamiento con HD convencional tres día por semana, con una duración media de 240 minutos (210-300 minutos), 26 con membranas de alta permeabilidad y 40 con membranas de baja permeabilidad. El flujo sanguíneo era 350 $\mathrm{ml} / \mathrm{min}$ y el flujo del baño $500 \mathrm{ml} / \mathrm{min}(\mathrm{n}=26)$ y $750 \mathrm{ml} / \mathrm{min}$. $(n=40)$. En cuanto al acceso vascular 48 tenían fístula autóloga y 18 fístula protésica (PTFE).

Se realizó un estudio prospectivo modificando el calibre de las agujas de punción, según el siguiente esquema:

1. Grupo 1: primera semana con aguja arterial $16 \mathrm{G}$ y venosa $15 \mathrm{G}$

2. Grupo 2: segunda semana con aguja arterial $15 \mathrm{G}$ y venosa $15 \mathrm{G}$

3. Grupo 3: tercera semana con aguja arterial $15 \mathrm{G}$ y venosa $14 \mathrm{G}$

Se extrajeron muestras de sangre de la aguja arterial al inicio y al final de la sesión tras 2' de bajar el flujo de sangre a 50 $\mathrm{ml} / \mathrm{min}$. Todas las sesiones se ajustaron según volumen de sangre pautado. En las muestras de sangre se midió la concentración de urea. El Kt/ V se determinó mediante la fórmula de Gotch y la de Daugirdas 2ํgeneración.
El dolor se cuantificó según una escala de valores: $0=$ sin dolor, $1=$ dolor leve, $2=$ dolor moderado y $3=$ dolor intenso.

Se registró el tiempo de coagulación en cada uno de los pacientes y se comparó con el habitual. Se consideró que el aumento del tiempo era: leve si se prolongaba de 1 a 10', moderado de 10 a 15' e intenso más de 15'. Se registraron los cambios aparecidos en la presión venosa y finalmente se anotaron aquellos casos en los que apareció sangrado periaguja.

Análisis estadístico: Se calcularon la media y la desviación estándar para cada valor. La comparación entre variables continuas se realizó mediante la prueba de "t" de Student pareada. La relación entre variables numéricas se determinó por análisis de correlación de Pearson. Las variables cualitativas se compararon mediante Chi-cuadrado y se utilizó también ANOVA para variables cuantitativas dependientes de un único factor. La hipótesis nula se rechazó en el análisis estadístico si p<0.05.

\section{RESULTADOS}

En la tabla 1 aparecen recogidas las modificaciones del $\mathrm{Kt} / \mathrm{V}$ tras el cambio de aguja. El uso de agujas $15 \mathrm{G}$ en la punción arterial indujo un aumento del $\mathrm{Kt} / \mathrm{V}$ estadísticamente significativo comparado con la punción con $16 \mathrm{G}$ como pone de manifiesto la diferencia encontrada entre el grupo 1 y los grupos 2 y 3 . En cambio el uso de agujas $14 \mathrm{G}$ en la punción venosa no alteró la cinética de la urea ya que no existió diferencia entre los grupos 2 y 3.

\begin{tabular}{|l|c|c|c|} 
& $\begin{array}{c}\text { Kt/V Todos } \\
(\mathbf{n = 6 6 )}\end{array}$ & $\begin{array}{c}\text { Kt/V Nativas } \\
(\mathbf{n = 4 8 )}\end{array}$ & $\begin{array}{c}\text { Kt/V Prótesis } \\
(\mathbf{n = 1 8 )}\end{array}$ \\
\hline Grupo 1 & $1.31(0.18)$ & $1.27(0.16)$ & $1.41(0.18)$ \\
\hline Grupo 2 & $1.37(0.16)^{*}$ & $1.35(0.14)^{*}$ & $1.41(0.2)$ \\
\hline Grupo 3 & $1.38(0.18)^{*}$ & $1.36(0.16)^{*}$ & $1.46(0.23)$ \\
\hline *p<0.001 entre grupo 1 y 2 y entre grupo 1 y 3 & \\
\hline
\end{tabular}

Tabla 1. Comparación entre Kt/ V (media $\pm D E)$ ) calculado en los diferentes grupos.

El segundo hallazgo relevante de nuestros datos es la diferencia que encontramos entre los portadores de fístula nativa y protésica, dado que las diferencias anteriormente mencionadas sólo se produjeron en los pacientes con acceso vascular autólogo sin que hubiera diferencia cuando se trataba de accesos protésicos.

Se hicieron distintos grupos separando los pacientes según el dializador utilizado y el tiempo de diálisis habitual sin que ninguno de estos factores mostrara ninguna influencia sobre el cambio de la aguja. 
En cuanto a las incidencias, al cambiar a la punción arterial con $15 \mathrm{G}$ no registramos ninguna. Sí que las encontramos en la punción venosa con 14G (grupo 3) que dio lugar a distintos eventos que quedan recogidos en la tabla 2. Además se produjo sangrado periaguja en 14 pacientes de los que 10 eran fístulas nativas y 4 protésicas.

\begin{tabular}{|l|c|c|c|c|}
\hline & \multicolumn{2}{|c|}{ Dolor } & \multicolumn{2}{c|}{ Tiempo coagulación } \\
\hline & Nativa & Prótesis & Nativa & Prótesis \\
\hline Leve & 10 & 3 & - & \\
\hline Moderado & 3 & 1 & 5 & 7 \\
\hline Intenso & 1 & 1 & 4 & 1 \\
\hline
\end{tabular}

Tabla 2. Número de pacientes que presentaron incidencias al utilizar agujas $14 \mathrm{G}$ en la punción venosa.

El estudio estadístico de estos datos mostró que existían diferencias significativas $(p<0.02)$ en la prolongación del tiempo de coagulación entre fístula nativa y protésica (figura 1) más no en la aparición de dolor ni de sangrado periaguja al utilizar 14 $G$ en la punción venosa.

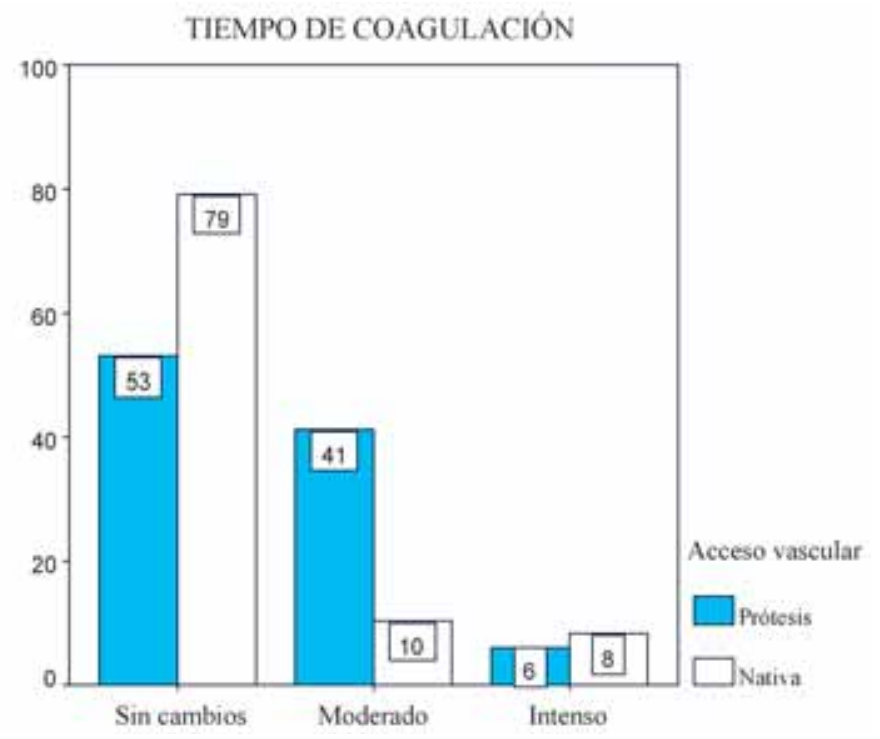

Figura 1. Diferencias en el tiempo de coagulación según acceso vascular.

Estudiamos a los pacientes que tenían una prolongación del tiempo de coagulación (moderado e intenso) y encontramos que la concentración de $\mathrm{Hb}$ era significativamente más baja en los que tenían una prolongación intensa que en los otros grupos y que, aunque los cambios no eran estadísticamente significativos, estos pacientes presentaban presiones venosas más elevadas ( $p>$ 0.07) y menor caída en la presión venosa en el paso de aguja 15G a 14G (Tabla 3). Dichos cambios fueron estadísticamente significativos sólo si se incluían a los pacientes con fístula nativa que tenían una PV más elevada que los que no habían presentado complicaciones $142.5 \pm 41.1$ vs $112.9 \pm 22.4 \mathrm{mmHg}$ $(p<0.02)$. Dichas diferencias ya existían con la aguja $15 \mathrm{G}$ $162.5 \pm 25.5$ vs. $140.2 \pm 21 \mathrm{mmHg}(p<0.02)$.

\begin{tabular}{|l|c|c|c|} 
& Sin cambios & Moderado & Intenso \\
\hline $\mathbf{H b}(\mathbf{g} / \mathbf{d L})$ & $12,1(1.2)$ & $12(1.1)$ & $10,5(0.7)^{*}$ \\
\hline $\mathbf{P V}(\mathbf{m m H g})$ & $119.6(26.2)$ & $127.5(32.3)$ & $144(35.7)$ \\
\hline $\mathbf{P V}$ (15G-14G) & $27.2(7.8)$ & $26.5(7)$ & $22(14.8)$ \\
\hline
\end{tabular}

$*_{p}<0.03$, entre intenso y los otros dos grupos

Tabla 3. Comparación según diferencias en tiempo de coagulación de la H b y PV. Media (DE).

\section{DISCUSIÓN}

Nuestro resultado demuestra que el aumento de calibre a una aguja 15G en la arteria permitió un aumento en la eficacia de la diálisis en las fístulas nativas, sin que se lograra mejorar en los accesos vasculares protésicos. Este hecho se debe a que al aumentar el calibre de la aguja a los flujos sanguíneos de bomba que nosotros utilizamos (350 $\mathrm{ml} / \mathrm{min}$ ) logramos aproximarnos a un flujo más real, lo que permite una mejoría en la depuración de urea.

Es un hecho conocido que el aumento del calibre de la aguja permite mayor flujo de sangre ${ }^{3,6}$ y que por tanto debe adecuarse la aguja al flujo que se trabaja. Pero no hemos encontrado en la bibliografía referencias sobre la influencia que pudiera tener el calibre de la aguja sobre la eficacia dependiendo del tipo de acceso vascular. En nuestros resultados la diferencia encontrada es importante, ya que la mejoría en la depuración de urea sólo la encontramos en las fístulas nativas. Aunque no contamos con medios para determinar el flujo del acceso vascular, el hallazgo nos hace suponer que los accesos protésicos tienen un mayor flujo que permiten conseguir flujos reales más altos que en las fístulas nativas y son menos dependientes del calibre usado. La detección de esta diferencia tiene importantes repercusiones desde el punto de vista práctico que lleva a una individualización de la pauta también en este aspecto.

El cambio a una aguja $14 \mathrm{G}$ en el retorno, a pesar de disminuir la presión venosa no mejora la eficacia de la diálisis en ningún tipo de acceso vascular. La base teórica que justificaría una posible mejoría es que la disminución de la presión venosa podría mejorar la recirculación. Nuestros resultados sin embargo muestran que la bajada de la presión venosa no conlleva una mejoría en el Kt/ V y que por lo tanto un flujo de sangre de $350 \mathrm{ml} / \mathrm{min}$ no es determinante de recirculación.

Además de los cambios favorables en la cinética de la urea el uso de las agujas $15 \mathrm{G}$ en la punción arterial no provocó ningún efecto secundario. Por el contrario, las punciones con 
agujas $14 \mathrm{G}$ condicionaron la aparición de dolor, mayor tiempo de coagulación y sangrado periaguja. El dolor fue considerado leve en la mayoría de los casos por lo que la mayor morbilidad era tolerable, pero no fue así con el tiempo de coagulación que se prolongó siempre en un grado considerado moderado o intenso. Es llamativo el hecho de que aunque globalmente se prolongara el tiempo de coagulación más en los accesos vasculares protésicos, el grupo de pacientes con sangrado intenso estuviera constituido sobre todo por portadores de fístulas nativas, que también presentaron más sangrado periaguja, aunque en este caso las diferencias no fueran estadísticamente significativas.

El análisis separado de los pacientes con fístula nativa y sangrado intenso $(n=5)$ demostró que presentaban presiones venosas más altas que el resto de los que tenían fístulas nativas, tanto con $14 \mathrm{G}$ como con $15 \mathrm{G}$. De éstos sólo uno tenía una estenosis central conocida, pero no podemos descartar que exista patología venosa en los otros que pudiera condicionar esta presión venosa más elevada y que se hiciera evidente esa prolongación en el tiempo de coagulación con una punción más traumática.

Por otra parte los que presentaron sangrado intenso (tanto fístulas nativas como prótesicas) tenían concentraciones de $\mathrm{Hb}$ más bajas. Podemos especular sobre la naturaleza de este hallazgo, que por un lado podría deberse a que un sangrado habitual prolongado fuera la causa de la anemia y por otro a que la propia anemia facilitara el sangrado. Por el pequeño grupo de enfermos y el diseño del trabajo no nos es posible definir la causa, pero este hallazgo merecería estudios más amplios que permitieran conocer si la concentración de $\mathrm{Hb}$ tiene alguna influencia en el tiempo de coagulación.

La principal limitación de nuestro trabajo es que no hemos medido el flujo real obtenido con el cambio de aguja. En cualquier caso existen datos bibliográficos suficientes que avalan que la mejoría del Kt/ V se debe a un flujo sanguíneo más próximo al flujo de la bomba. Considerando el punto de vista práctico, la medida del flujo de sangre no es posible en muchas unidades de HD y lo que es más importante es lograr la mejor depuración de urea con prácticas que no impliquen morbilidad al paciente y sean económicamente rentables. A la vista de nuestros resultados podemos decir que para que el calibre sea el ideal, habría que adecuarlo a los flujos de sangre que utilizan en cada unidad de diálisis y al tipo de acceso vascular, quedando abierta la posibilidad de más estudios con diferentes tamaños de agujas y flujos de sangre.

En resumen, los resultados de nuestro trabajo nos permiten recomendar el uso de aguja $15 \mathrm{G}$ en arteria y vena de fístulas nativas para que conseguir una mejor eficacia en la HD a flujo de sangre de $350 \mathrm{~mL} / \mathrm{min}$, sin aumento del coste y sin generar morbilidad para el paciente. Por otra parte la utilización de aguja $14 \mathrm{G}$ en la punción venosa produce la aparición de efec- tos secundarios y no mejora la cinética de la urea por lo que su uso no nos parece indicado.

\section{CONCLUSIONES}

- El estudio demuestra que en pacientes con fístula nativa el aumento del calibre de la aguja arterial de 16 G a $15 \mathrm{G}$ mejora de forma significativa la eficacia de la diálisis, sin embargo no se encontraron diferencias en pacientes con fístulas protésicas. Por otra parte, el uso de la aguja $14 \mathrm{G}$ en la punción venosa no indujo mejoría significativa en el Kt/ $\mathrm{V}$ en ningún tipo de acceso vascular.

- En cuanto a otros efectos del cambio de aguja no existieron problemas en el paso de 16G a 15G. Sí que el uso de aguja $14 \mathrm{G}$ produjo más episodios de dolor, de sangrado periaguja y una prolongación de tiempo de sangrado que fue más frecuente en pacientes con fístula nativa que tenían presiones venosas más elevadas.

- Los pacientes que tenían un aumento en el tiempo de coagulación tenían una concentración hemoglobina más baja.

- Nuestros resultados apoyan por tanto el uso de agujas 15G en la punción arterial de las fístulas nativas cuando se dializa a un flujo de sangre de $350 \mathrm{~mL} / \mathrm{min}$; con ello se consigue mejorar la depuración al mejorar el flujo de sangre real sin que aparezcan efectos deletéreos. Dicho beneficio no se produce en las fístulas protésicas, probablemente debido a que tienen un mayor flujo de sangre y la influencia del calibre utilizando flujos menores de $400 \mathrm{~mL} / \mathrm{min}$ no se pone de manifiesto en estos accesos.

\section{AGRADECIMIENTOS}

Queremos agradecer la colaboración de todas nuestras compañeras, a Pilar Mayo y a nuestra supervisora Concepción Andrea por el apoyo prestado.

\section{BIBLIOGRAFÍA}

1. Daugirdas J T. Manual de diálisis. Barcelona: Masson; 2003.

2. Lorenzo V. Manual de nefrología clínica. Madrid: Harcourt Brace; 1998.

3. Gallego $E$ et al. Efecto del calibre de las agujas sobre la recirculación y la eficacia de la HD. Nefrología 1997; 16:322.

4. Sánchez Villar I et al. Calibre de la aguja y calidad de la hemodiálisis. Comunicación en el XXIV Congreso de la SEDEN. Valencia. 1999; 120-124.

5. Tovar A et al. Efecto del cambio de aguja sobre la eficacia de la hemodiálisis, las presiones del circuito y la recirculación. Comunicación en el XXI Congreso de la SEDEN. Salamanca. 1996; 204-210.

6. Hitesh K et al. Correction of discrepancy between prescribed and actual blood flow rates in chronic haemodialysis patients with use of larger gauge needles. Am J Kidney Dis 2002, 39:1231. 